\title{
Celiac Disease Diagnosed using Video Capsule Endoscopy in a Patient with Crohn's Disease
}

A 44-year-old woman with fluctuating iron deficiency developed anemia, abdominal discomfort, weight loss and diarrhea 1 year prior to admission for video capsule endoscopy (VCE), which is the method of choice in the work-up of suspected disease in the small intestine [1]. The patient had undergone a total colectomy, 20 years earlier, due to histologically confirmed Crohn's disease and had received a permanent ileostomy.

Based on the patient's history and present symptoms, the admitting gastroenterologist suspected a relapse of Crohn's disease and scheduled the patient for immunosuppressive treatment with azathioprine. To rule out stricture, a small-bowel enteroclysis was conducted but yielded no suspected disease in the remaining intestine.

VCE was performed after an overnight fast, without further bowel preparation and with 8 hours of ambulatory data registration. The VCE examination revealed mucosal changes (scalloped folds and mosaic patterning) in the duodenum and jejunum, suggesting celiac disease (Figure 1). The ileal mucosa was normal with no signs of recurrent Crohn's disease. These findings were confirmed by a subsequent duodenoscopy combined with chromoendoscopy (Figure 2 ) and ileoscopy. Histological examination confirmed mucosal villous atrophy in the distal duodenum (Figure 3), whereas the ileal mucosa was normal. Celiac disease was also confirmed by serology (a positive serum antiendomysial antibody test). Accordingly, the patient was put on a glutenfree diet and is now doing well without any gastrointestinal symptoms.

Intensive investigative effort is currently focused on defining the potential role of VCE in clinical practice. This case illustrates that VCE may play a key role in the differential diagnosis of diseases emanating from the small intestine. In fact, our case shows that VCE was not only important for the diagnosis but also affected the

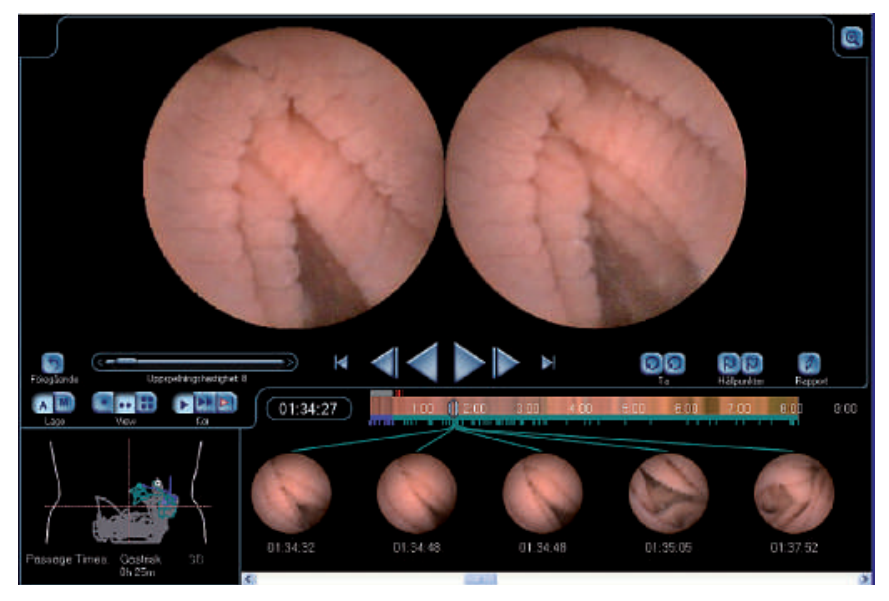

Figure 1 Video capsule endoscopy findings, showing scalloped folds and a mosaic pattern that suggest celiac disease in the proximal small bowel.

management of this patient with celiac disease.

\section{E. Toth' ${ }^{1}$ B. Ohlsson ${ }^{1}$, O. Ljungberg ${ }^{2}$,}

\section{H. Thorlacius ${ }^{3}$}

${ }^{1}$ Department of Medicine, Malmö University Hospital, Malmö, Lund University, Sweden

2 Department of Pathology, Malmö University Hospital, Malmö, Lund University, Sweden

${ }^{3}$ Department of Surgery, Malmö University Hospital, Malmö, Lund University, Sweden.

\section{References}

${ }^{1}$ Gay G, Delvaux M, Rey JF. The role of video capsule endoscopy in the diagnosis of digestive diseases: a review of current possibilities. Endoscopy 2004; 36: 913 -920

\section{Corresponding Author}

\section{E. Toth, M.D., PhD}

Department of Medicine

Endoscopy Unit

Malmö University Hospital

Lund University

S-205 02 Malmö

Sweden

Fax: $\quad+46-40-338699$

E-mail: ervin.toth@med.lu.se

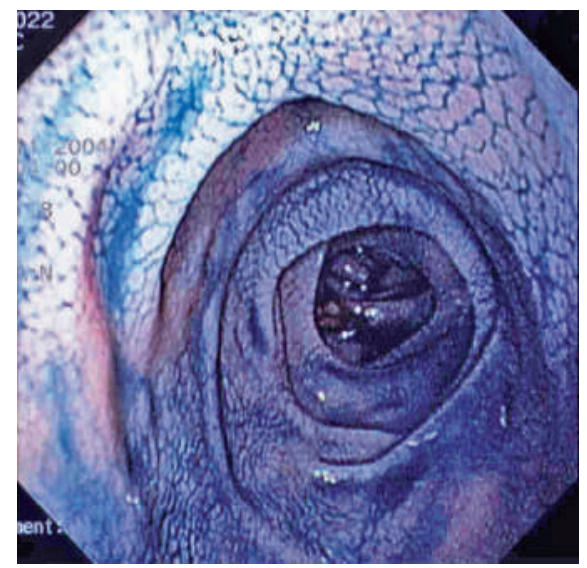

Figure 2 A gastroscopic image after dye spraying with indigo carmine, shows loose and scalloping of mucosal folds with mosaic patterning in the duodenum.

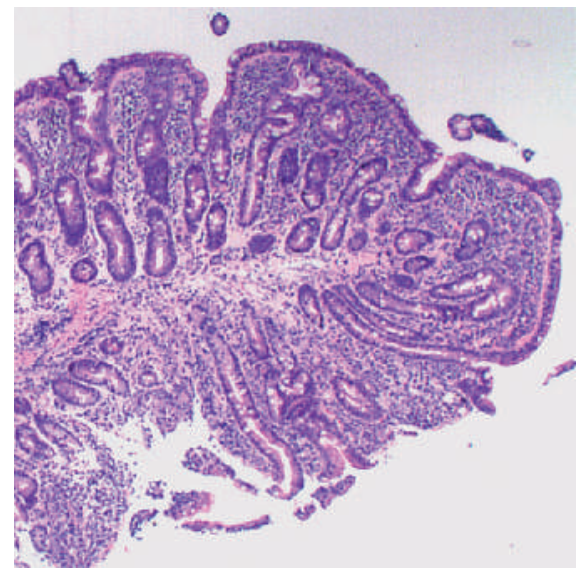

Figure 3 Microphotograph of duodenum, demonstrating marked villous atrophy. 